\title{
Hereditary twenty-nail dystrophy in a Sicilian family
}

\author{
L PAVONE, S LI VOLTI, B GUARNERI*, M LA ROSA, G SORGE, \\ G INCORPORA, AND F MOLLICA
}

From the Departments of Paediatrics and * Dermatology, University of Catania, Catania, Italy

SUMMARY Over three successive generations four members of a Sicilian family showed early onset, slowly progressive dystrophy of the finger and toe nails, without any other anomalies or skin signs. Histological examination performed in two patients enabled us to discount lichen planus and other underlying diseases. The hereditary and idiopathic nature of the disease is underlined and a classification of the dystrophies affecting all the 20 nails is proposed.

In 1977 Hazelrigg et $a l^{1}$ described 'twenty-nail dystrophy of childhood', an apparently idiopathic disease characterised by dystrophic changes of all 20 nails in children entirely free of other skin, hair, and teeth abnormalities. The idiopathic nature of this disorder has been questioned by other workers, who believe it is an expression of alopecia $\operatorname{areata}^{2} 3$ or lichen planus ${ }^{4}$ confined to the nails.

Even if most cases of this disease are sporadic, some families have been reported in whom a clinically identical disease to that described by Hazelrigg $e t$ al $^{1}$ was transmitted through successive generations with an autosomal dominant pattern of inheritance. $^{5-8}$

In this paper we report a family with four affected members in three successive generations. Nail biopsy was performed in two patients. Our results suggest that a hereditary form of twenty-nail dystrophy exists and is a distinct clinical entity.

\section{Case reports}

THE PROBAND

The proband (fig 1, III.2), a 17-year-old male farmer, was admitted to the Dermatology Department of the University of Catania because of nail loss (fig 2). At birth his parents had noticed that his nails were uniformly thin and opalescent with longitudinal striations. Lamellar splitting of all nails occurred over the following years until there was almost complete nail loss at 10 years. Apart from the nails, his past history was unremarkable. On examination there were no signs of eczema, lichen planus, or psoriasis on the skin or mucous membranes. Hair and teeth were also normal. No alterations of the patellae or other bones were present. Cultures for dermatophytes and yeasts were repeatedly negative.

Received for publication 15 January 1982

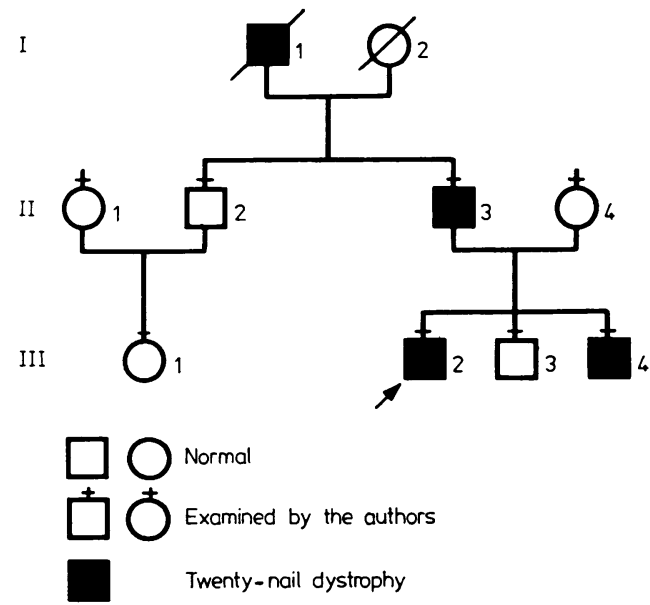

FIG 1 Family pedigree.

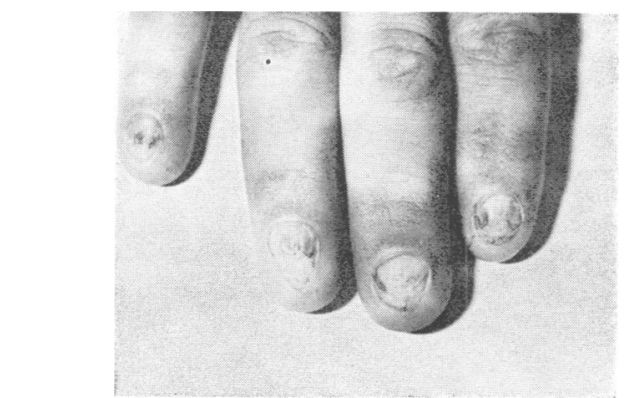

FIG 2 Proband's fingers.

THE FAMILY

The family lived in Vittoria, a small town in southeastern Sicily. Information about the family was obtained from II.3. We examined all eight living subjects shown in the pedigree. 


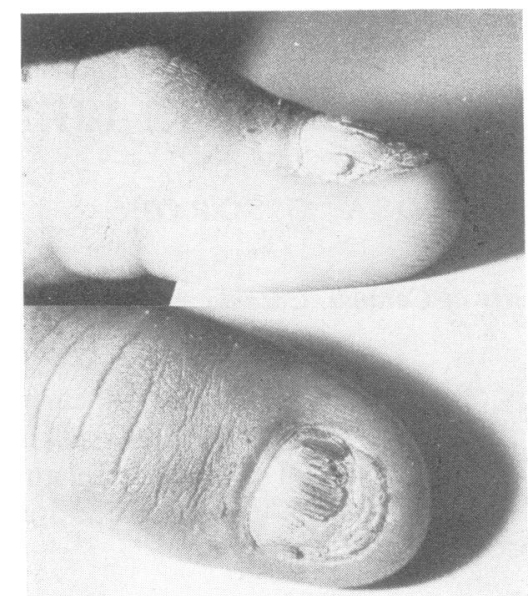

FIG 3 Father's finger (lateral and dorsal views).

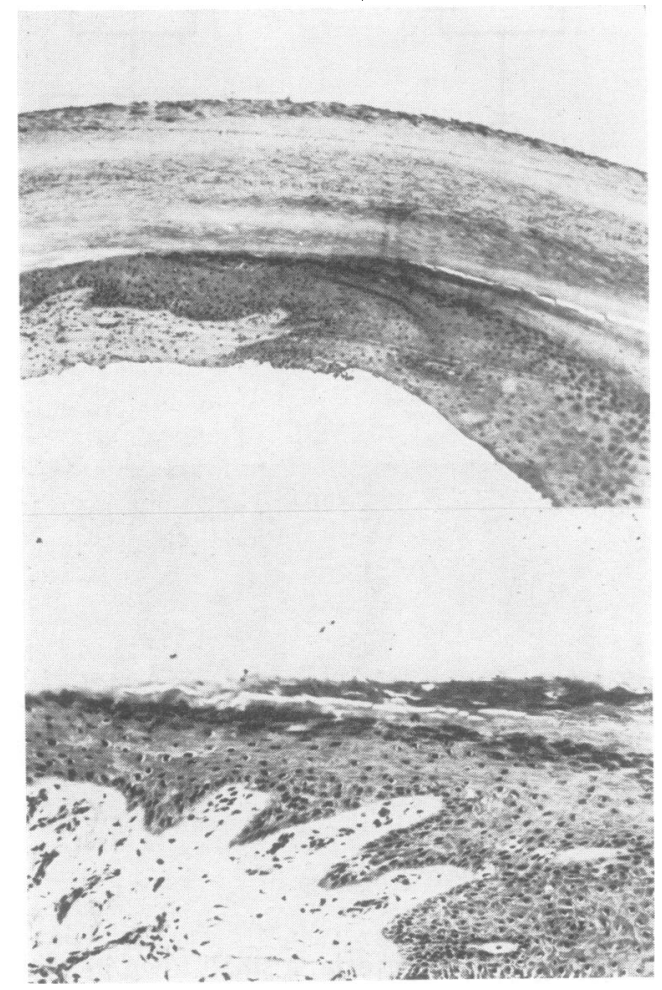

FIG 4 Proband's nail biopsy. Original magnification $\times 50($ top $)$ and $\times 125$ (bottom).
I.1, a male farmer who died at the age of 77 years, was said to have had no nails. We were told that his nails had been thin since birth and that he had lost them at the age of 20 . There was no reliable information about his parents.

II.3, the proband's father, was a farmer aged 41. His nails had been opalescent with longitudinal ridging and with easily broken edges from birth. The alterations were more evident after puberty (fig 3). On examination no signs of nail fold involvement or other dermatological or skeletal disease were present. Cultures for dermatophytes and yeasts were repeatedly negative.

III.4, the proband's brother, was a 7-year-old schoolboy. His finger and toe nails showed longitudinal ridging and distal notching, and were thin and friable. There was no history of eczema, lichen planus, or any other skin diseases. On examination, his hair, buccal mucosa, teeth, and skeleton, including the patellae, were normal. Cultures for fungi and yeasts were repeatedly negative.

\section{HISTOLOGICAL STUDY}

Biopsy specimens of the nail bed and plate were obtained from the lateral portion of the left forefinger of the patient (III.2) and his father (II.3).

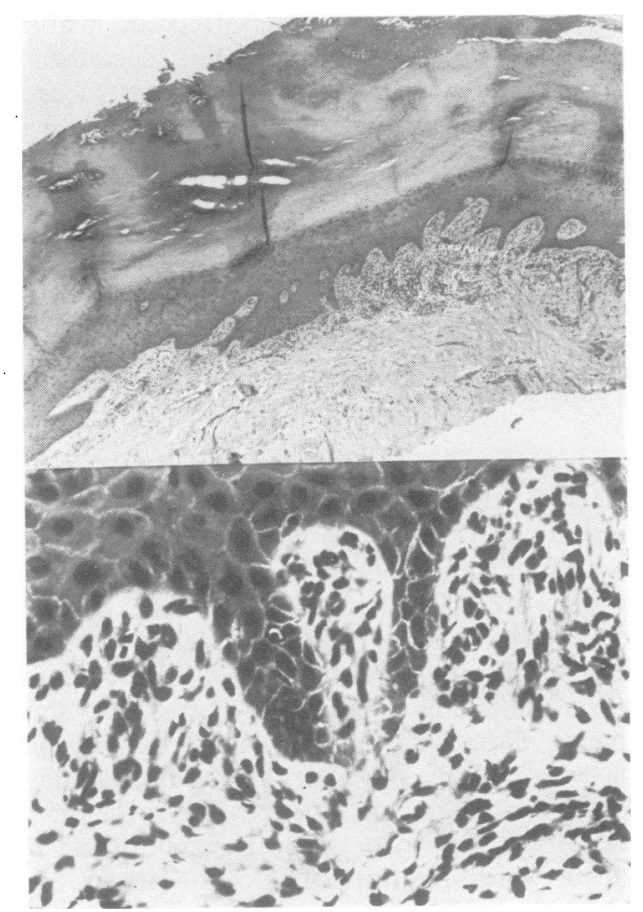

FIG 5 Father's nail biopsy. Original magnification $\times 30$ (top) and $\times 312$ (bottom). 
III.2. Histological examination (fig 4) revealed epidermal thickening with acanthosis, and a multilayered stratum granulosum. There was splitting of the stratum lucidum. The nail bed epidermis was thin with flattened germinative buds. Some degree of vascular proliferation was present in the nail bed dermis. Mild degenerative changes were noted in the external and central layers of the dermal collagen, where there were very few reticular fibres. No signs of lichen planus were seen.

II.3. The histological picture (fig 5) was very similar to that of III.2, but the degenerative changes of the dermis were more evident. An additional feature was the marked mononuclear infiltration of the superficial dermis. In some areas this infiltration came into contact with the epidermis without disrupting its basal layer.

\section{Discussion}

The features of our patients were the following: (1) involvement of all nails; (2) family history; (3) slow progression; and (4) absence of peculiar histological features. The anomaly present in this family affected four members in three successive generations and showed a dominant pattern of inheritance.

The absence of associated developmental defects allowed us to rule out three other dominantly inherited diseases characterised by dystrophic changes of the nails similar to those in our patients. These are (1) the nail patella syndrome, because of the lack of abnormalities of the patellae and the other bones; (2) pachyonychia congenita, because there was no palmar and plantar hyperkeratosis, follicular keratosis of the skin, leucoplakia, or other ungual lesions; and (3) hidrotic ectodermal dysplasia, because none of our patients had alopecia or hyperpigmented skin. In 1928, Jacobsen ${ }^{9}$ reported families in which many members had nail dystrophy and alopecia. They probably had hidrotic ectodermal dysplasia.

Nail involvement is also found in some nonMendelian skin diseases. Nails are involved in seven to $66 \%$ of patients with alopecia areata. ${ }^{3}$ Baran et $a l^{3}$ found alopecia areata in five out of six patients with nail dystrophy and thought that the apparently idiopathic twenty-nail dystrophy might actually be a manifestation of alopecia areata. However, it must be stressed that alopecia areata is a non-hereditary disease and that none of our patients had hair abnormalities or had a past history of alopecia.

The nail changes that may be found in psoriasis are quite different from those seen in our patients. They consist mainly of onycholysis and pitting of the nail plate, which resembles a thimble at times. The plate may be opaque, thickened at its distal edge, and detached so that the finger assumes a rush-pith appearance. Approximately one to $10 \%$ of patients with lichen planus also show dystrophic changes of the nails. These vary from slight roughening and ridging of the nail surface to total destruction and atrophy. ${ }^{10}$ Even if lesions are rare in childhood and are usually accompanied by typical lichen planus of the skin elsewhere, ${ }^{10}$ a variety of lichen confined to the nails has been reported.11 Moreover, unequivocal histological signs of lichen planus were found in two unrelated patients with isolated nail dystrophy. ${ }^{4-10}$ It has therefore been suggested that twenty-nail dystrophy is actually a variant of lichen planus. ${ }^{4} \mathrm{We}$ do not think that this interpretation may be applied to our patients for the following reasons.

(1) None of the most common signs of nail lichen planus (longitudinal ridges and furrows, thinning of nail plate, pterygium, violet lines or papules in the nail bed visible through the nail plate, severe inflammatory reaction of the nail matrix and bed) ${ }^{10}$ was present in our patients.

(2) The lesions of lichen planus rarely involve all the toe and finger nails, while each of our patients was affected in all 20 nails.

(3) Lichen planus is a non-familial and non-hereditary disease, while the disease reported here is inherited as an autosomal dominant trait.

However, only nail biopsy could exclude the possibility of a very unusual variety of lichen planus in our patients. Nail biopsy has not been carried out on any of the familial cases of twenty-nail dystrophy described so far. We performed a nail bed and plate biopsy in two of the four affected members of the family, the 17-year-old proband and his father. Our proband showed non-specific changes of the epidermis (irregularly thickened stratum granulosum, flattened germinative buds) and of the dermis (some degree of vascular proliferation, mild degenerative changes), but no signs of lichen planus. In the father, the degenerative lesions of the dermis were more evident and reactive mononuclear infiltrates of lichenoid appearance were present. These infiltrates had no typical features of nail lichen planus (massive hyperkeratosis of the nail bed, basal cell degeneration with disorders of pigment, band-like infiltrate hugging the epidermis and disrupting its basal layer).

Many of the reported cases of twenty-nail dystrophy may well fall within one of the above mentioned diagnoses. The apparently idiopathic cases are less frequent and the familial cases are exceedingly rare..$^{5-8}$ Moreover, none of the latter has been confirmed by biopsy. In this report the existence of a 
hereditary variety of twenty-nail dystrophy independent from other skin disease is documented for the first time.

The natural course of this disease deserves attention. Hazelrigg et $a l^{1}$ suggested that twentynail dystrophy may be a self-limiting abnormality which slowly improves with age. This does not agree with our data and with some of the cases reported. ${ }^{12}$ Subject $\mathbf{I . 1}$ in our pedigree lost his nails almost completely in his twenties and did not regain them before he died at 77 . Also his son showed severe nail alterations about 25 years ago and since then the anomaly has been slowly worsening. This suggests that hereditary twenty-nail dystrophy is a slow and inevitably progressive disease, even if the only discomfort it causes is the cosmetic aspect.

At present we feel that there are many forms of

TABLE A proposed classification of dystrophies affecting all 20 nails

\begin{tabular}{lll}
\hline & $\begin{array}{l}\text { Associated with } \\
\text { other disorders }\end{array}$ & Isolated \\
\hline Congenital & $\begin{array}{l}\text { Nail patella syndrome } \\
\text { Pachyonychia congenita } \\
\text { Hidrotic ectodermal } \\
\text { dysplasia }\end{array}$ & $\begin{array}{l}\text { 'TND' of childhood } \\
\text { (present family) } \\
\text { Acquired }\end{array}$ \\
$\begin{array}{l}\text { Lichen planus } \\
\text { Alopecia areata } \\
\text { Psoriasis }\end{array}$ \\
\hline
\end{tabular}

dystrophy involving all 20 nails, which could be classified as in the table.

\section{References}

1 Hazelrigg DE, Duncan WC, Jarratt M. Twenty-nai dystrophy of childhood. Arch Dermatol 1977;113:73-5.

2 Baran R, Dupré A. Vertical striated sandpaper nails. Arch Dermatol 1977;113:1613.

3 Baran R, Dupré A, Christol B, Bonafé JL, Sayag J, Ferrère J. L'ongle grésé peladique. Ann Dermatol Venereol $1978 ; 105: 387-92$.

${ }^{4}$ Scher R, Fischbein R, Ackerman AB. Twenty-nail dystrophy. A variant of lichen planus. Arch Dermatol 1978;114:612-3.

5 Pires de Lima JA. Onychatrophie familiale congénitale. Ann Dermatol Syph 1924;6:266-71.

6 Tobias N. Hereditary familial dystrophy of the nails. JAMA 1925;84:1568-9.

7 Thompson HB. Hereditary dystrophy of the nails. JAMA 1928;91:1547.

8 Achten G, Wanet-Rouard J. Atrophie unguéale et trachyonychie. Arch Belg Dermatol 1974;30:201-7.

9 Jacobsen AW. Hereditary dystrophy of the hair and nails. JAMA 1928;90:686-9.

10 Zaias N. The nail in lichen planus. Arch Dermatol 1970; 101:264-71.

11 Burgoon CF Jr, Kostrzewa RM. Lichen planus limited to the nails. Arch Dermatol 1969;100:371.

12 Synkowski DR. Twenty-nail dystrophy. Arch Dermatol 1977;113:1462.

Requests for reprints to Professor Florindo Mollica, Clinica Pediatrica, Città Universitaria, Viale A Doria 6, Catania 95125, Italy. 